\title{
Development of a Confined Circular-cum-parallel Ribbon Flare and Associated Pre-flare Activity
}

\author{
Pooja Devi ${ }^{1}$ - Bhuwan Joshi ${ }^{2}$. \\ Ramesh Chandra ${ }^{1}$. Prabir K. Mitra ${ }^{2}$. \\ Astrid M. Veronig ${ }^{3}$ - Reetika Joshi ${ }^{1}$
}

(c) Springer $\bullet \bullet \bullet \bullet$

\begin{abstract}
We study a complex GOES M1.1 circular ribbon flare and related preflare activity on 26 January 2015 [SOL2015-01-26T16:53] in solar active region NOAA 12268. This flare activity was observed by the Atmospheric Imaging Assembly (AIA) on board Solar Dynamics Observatory (SDO) and the Reuven Ramaty High Energy Solar Spectroscopic Imager (RHESSI). The examination of photospheric magnetograms during the extended period, prior to the event, suggests the successive development of a so-called "anemone" type magnetic configuration. The Nonlinear Force Free Field (NLFFF) extrapolation reveals a fan-spine magnetic configuration with the presence of a coronal null-point. We found that the pre-flare activity in the active region starts $\approx 15 \mathrm{~min}$ prior to the main flare in the form of localized bright patches at two locations. A comparison of locations and spatial structures of the pre-flare activity with magnetic configuration of the corresponding region suggests onset of magnetic reconnection at the null-point along with the low-atmosphere magnetic reconnection caused by the emergence and the cancellation of the magnetic flux. The main flare of M1.1 class is characterized by the formation of a well-developed circular ribbon along with a region of remote brightening. Remarkably, a set of relatively compact parallel ribbons formed inside the periphery of the circular ribbon which developed lateral to the brightest part of the circular ribbon. During the peak phase of the flare, a coronal jet is observed at the north-east edge of the circular ribbon which suggests interchange reconnection between large-scale field lines and low-lying closed field lines. Our investigation suggests a combination of two distinct processes in which ongoing pre-flare null-point reconnection gets further
\end{abstract}

$\triangle$ Pooja Devi

setiapooja.ps@gmail.com

1 Department of Physics, DSB Campus, Kumaun University, Nainital 263002, India

2 Udaipur Solar Observatory, Physical Research Laboratory, Udaipur 313004, India

3 Institute of Physics \& Kanzelhöhe Observatory for Solar and Environmental Research, University of Graz, A-8010 Graz, Austria 
intensified as the confined eruption along with jet activity proceeded from within the circular ribbon region which results to the formation of inner parallel ribbons and corresponding post-reconnection arcade.

Keywords: Sun - flares: Sun - corona: Sun - jets: Sun - magnetic fields

\section{Introduction}

Solar flares are the result of a sudden release of free magnetic energy stored in active regions (Parker, 1963, Fletcher et al., 2011; Shibata and Magara, 2011). However, there are also the cases where flares can occur outside the active region (for example Chandra et al., 2017). Broadly they can be classified in two categories i.e. eruptive and confined flares (Svestka, 1986). Eruptive flares are usually long duration two-ribbon flares that show prolonged energy release (up to several hours). These type of flares occur in association with coronal mass ejections (CMEs). In contrary, confined flares are short duration flares showing localized brightening and are not accompanied by CMEs (for detail see the review by Shibata and Magara, 2011).

According to the morphology, a new category of flares have been reported which are known as circular ribbon flares (Wang and Liu, 2012). These flares usually contain a circular or quasi-circular ribbons and an inner ribbon inside it. Circular ribbon flares can be confined (Masson et al., 2009, Zuccarello et al., 2017. Hernandez-Perez et al., 2017) as well as eruptive in nature (Hong et al., 2017, Li et al., 2018). According to the magnetic topology, circular ribbon flares occur in anemone active regions (Asai et al., 2008). Anemone active regions are specified as active regions, where a single polarity is surrounded by opposite polarity in a circular pattern. In some of the circular ribbon flares, one remote ribbon is present and it normally appears after the formation of the circular ribbon. However, there are also cases where the remote ribbon appears simultaneously with the circular ribbon. Another important ingredient, which has been observed in many circular ribbon flares, is the presence of jets/surges at the boundary of the circular ribbon. The combination of circular and remote ribbons along with jets/surges were also studied in the numerical simulations (Masson et al., 2009 Pariat, Antiochos, and DeVore, 2010). According to the literature, the first detailed study of a circular ribbon flare was presented by Masson et al. (2009). Since then circular ribbon flares have received quite some attention which resulted in many subsequent studies (e.g., Reid et al., 2012, Wang and Liu, 2012; Sun et al., 2013, Jiang and Feng, 2013, Xu et al., 2017; Hernandez-Perez et al., 2017). Very recently, Hao et al. (2017) reported white light kernels in a circular ribbon flare. Further, with high resolution IRIS data, the characteristics of two circular ribbon flares were studied ( $\mathrm{Li}$ et al., 2018).

To explain the general characteristics of eruptive solar flares including the evolution of flare ribbons and loops, the standard CSHKP model was put forward by Carmichael (1964), Sturrock (1966), Hirayama (1974), and Kopp and Pneuman (1976). However, this 2-Dimensional model does not explain the formation of circular flare ribbons. Recently, Aulanier, Janvier, and Schmieder (2012) 
extended the 2D model into 3D (see also the review by Janvier, Aulanier, and Démoulin, 2015). Circular ribbon flares provide us an opportunity to understand the flare in 3D because they are related to magnetic field topology of fan-spine or 3D null-point magnetic topology in the corona. Although many circular ribbon flares have been reported, there are still several questions about the origin of such flares, such as, why only some flares have remote ribbons together with circular ribbon?; What is the temporal relation between circular and remote ribbons?; Why are jets/surges not always present in all the cases?; Why only few anemone active region produce such type of flares?; etc.

With reference to the above questions, we present here a study of a circular ribbon flare of GOES class M1.1 observed on 26 January 2015 using multi-instrument data. An important aspect of present observations is the identification of distinct pre-flare activity which initiates $\approx 15$ min prior to the circular ribbon flare. The flaring site exhibits classical fan-spine configuration with a coronal null-point while the underlying photospheric fields shows "anemone" type morphology at the site of fan-dome. The main phase of the flare is characterized by the formation of circular ribbon and subsequent remote ribbon with fine-structured kernels. Afterwards, the circular ribbon evolves into the parallel ribbons with the formation of a post-flare loop between a set of conjugate (parallel) ribbons. Further important finding includes the apparent progression of brightness at remote ribbon location which we attribute to the slipping reconnection. The paper is organized as follows: Section 2 provides the information of the data sets used for the investigation. The analysis and results of the event based on EUV/UV and X-ray observations are given in Section 3. Finally, we discuss and conclude our results in Section 4.

\section{Observational Data Sets}

For the current study, we used the data from following sources:

- SDO/AIA Data: The Atmospheric Imaging Assembly (AIA: Lemen et al., 2012) on board the Solar Dynamics Observatory (SDO: Pesnell, Thompson, and Chamberlin, 2012) observes the different layers of the Sun in seven EUV $(94 \AA(6 \mathrm{MK}), 131 \AA(10 \mathrm{MK}), 171 \AA(600,000 \mathrm{~K}), 193 \AA$ ( $1 \mathrm{MK}), 211 \AA(2$ $\mathrm{MK}), 304 \AA(50,000 \mathrm{~K})$, and $335 \AA(2.5 \mathrm{MK}))$, two UV $(1600 \AA(10,000 \mathrm{~K})$, and $1700 \AA(4500 \mathrm{~K})$ ), and one White-light (4500 $\AA$ (6000 K)) channels with a cadence of 12 second, 24 second, and 3600 second, respectively. The pixel size of AIA data is $0.6^{\prime \prime}$. In this paper, we have analyzed AIA images in 94 $\AA, 171 \AA, 304 \AA$, and $1600 \AA$ wavelengths to study the flare in the corona, upper transition region, chromosphere, and photosphere, respectively.

- SDO/HMI Data: The Helioseismic Magnetic Imager (HMI: Schou et al., 2012) on board SDO observe the full disk of the Sun at $6173 \AA$ with a spatial resolution of $1^{\prime \prime}$. Here, we use LOS magnetic field maps, vector magnetograms, and white light data of HMI to study the magnetic structure and sunspot distribution of the active region, respectively. 
- RHESSI Data: The Reuven Ramaty High Energy Solar Spectroscopic Imager (RHESSI: Lin et al., 2002) performs imaging and spectroscopy of Xray and gamma-rays emitted during solar flares. RHESSI observes the full disk of the Sun with a spatial resolution as fine as $2.3^{\prime \prime}$ (energy range from $3 \mathrm{keV}$ to $20 \mathrm{MeV}$ ). We reconstructed RHESSI imaging using the CLEAN algorithm. We have used all 9 front detector segments except 2 and 7 to reconstruct the images. The spatial sampling of the reconstructed images is $2.0^{\prime \prime}$ per pixel.

- GONG Data: The Global Oscillation Network Group (GONG: Harvey et al., 2011) provides full disk $\mathrm{H} \alpha$ filtergram of the Sun. The temporal and spatial resolutions are $1 \mathrm{~min}$ and $2^{\prime \prime}$, respectively.

\section{Analysis and Results}

Active region NOAA 12268 emerged on the solar disk on 23 January 2015 at the heliographic location S10E67 with a $\beta$ type of magnetic configuration. As time progressed, the magnetic configuration of the active region changed to more complex $\beta \gamma$ type. The active region completed its journey on the solar disk on 04 February 2015 and its magnetic configuration remained $\beta \gamma$.

On 26 January 2015, the active region produced a GOES flares of class M1.1 (peak time $\approx 16: 53 \mathrm{UT}$ ). On that day, the active region was located at S10E35 on the solar disk. The overview of the active region observed by SDO in different channels and $\mathrm{H} \alpha$ by GONG is presented in Figure 1. The active region consisted of a large leading sunspot of positive polarity followed by trailing sunspot groups of mixed polarity region with predominantly negative polarity. Since the flares occurred in the following polarity region, the location of the main flare was at the region where positive magnetic polarity was surrounded by negative magnetic polarity. The active region was also very active during 29-30 January 2015 and produced six flares of moderate intensities (Zhong et al., 2019).

According to the GOES X-ray observation, the flare started $\approx 16: 46 \mathrm{UT}$, peaked at $\approx 16: 53 \mathrm{UT}$ and ended at $\approx 16: 58 \mathrm{UT}$. The flare was compact in nature and not associated with a filament eruption or CME. However, during the flare, jet activity was observed, suggesting a confined eruption along largescale field lines. Figure 2 displays the temporal evolution of the flare in X-rays and EUV/UV wavelengths. The time profiles can be divided into four parts namely, pre-flare phase, impulsive phase, maximum phase, and decay phase. The description of these phases are presented as follows:

The pre-flare phase (between 16:32-16:48 UT) is visible in the GOES 0.5-4 $\AA$ channel of and RHESSI $3-6$ and 6-12 keV energy bands along with several AIA EUV/UV (1600, 304, 193, 171, and $94 \AA$ ) wavelengths (see Figure 22). The impulsive phase (between 16:48-16:50 UT) of the flare starts $\approx 4$ min earlier in the RHESSI lower energy bands $(3-6$ and $6-12 \mathrm{keV})$ in comparison to higher energy bands ( $12-25$ and $25-50 \mathrm{keV}$ ). The maximum phase (starting from $\approx 16: 50$ UT) shows two peaks at 16:51 UT and 16:58 UT in all the RHESSI energy bands and in some of the AIA EUV channels (193 and $171 \AA$ ). Here it is to be noted 


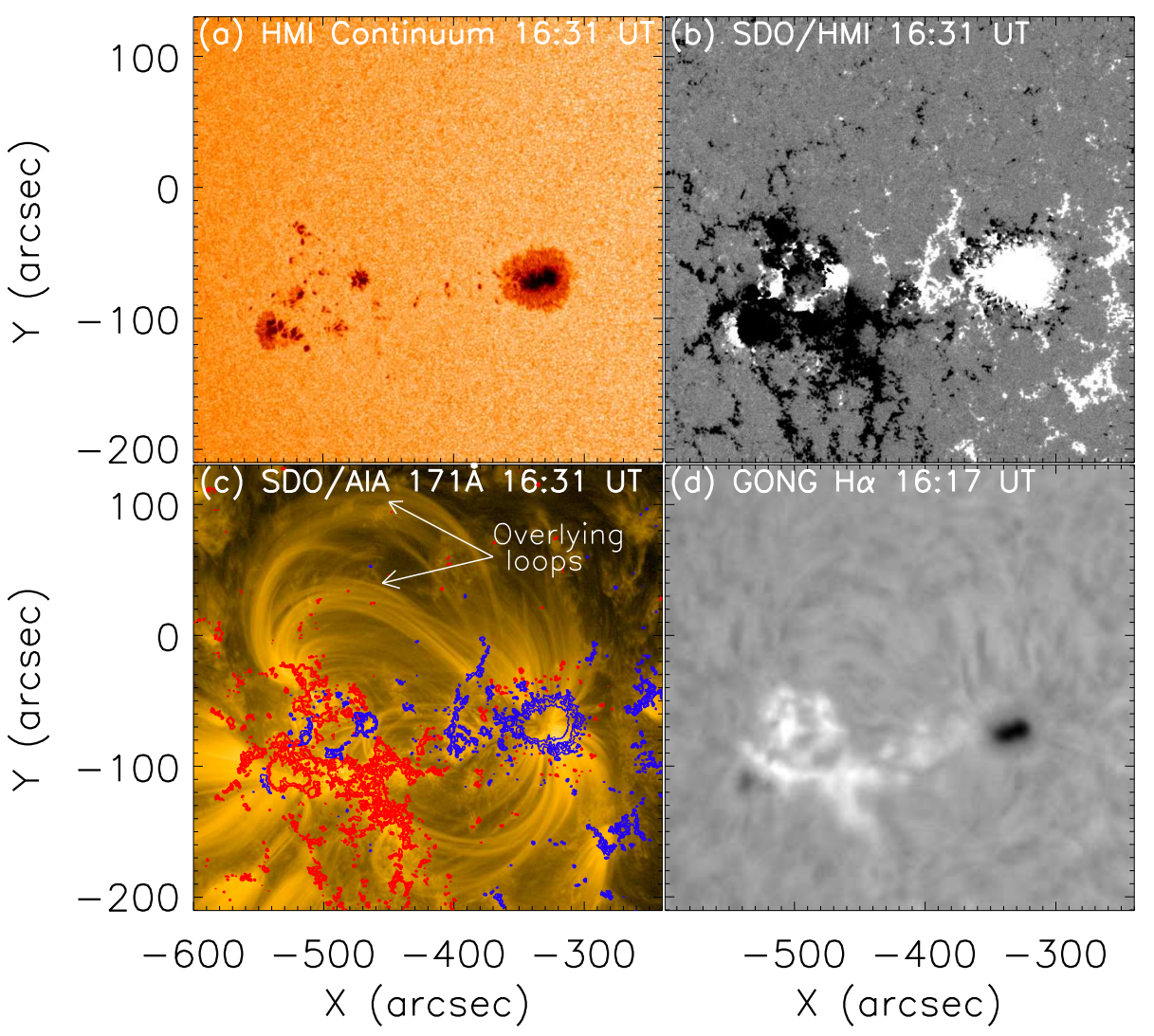

Figure 1. Multi-wavelength view of NOAA AR 12268. HMI continuum (a) and HMI magnetogram (b) showing that the leading sunspot have positive magnetic polarity and multiple smaller trailing sunspots have mixed (positive and negative) magnetic polarity. The AIA 171 $\AA$ image with over-plotted magnetic field is shown in (c). Blue and red contours corresponds to positive and negative magnetic polarities, respectively. The levels of these contours are \pm 150 , \pm 300 , and \pm 600 G, respectively. The GONG H $\alpha$ image is shown in (d).

that AIA 94, 304, and $1600 \AA$ shows only one peak. The peak of 304 and $1600 \AA$ are co-temporal with the first peak, whereas AIA $94 \AA$ coincides with the second peak time. After maximum, the decay phase of the flare starts from $\approx 17: 00 \mathrm{UT}$ onward, showing a gradual decrease.

\subsection{Pre-flare Activity}

The pre-flare phase is defined between 16:32 UT and 16:48 UT. We found slight increase in the intensity of the flaring region during this period. Figures $3 \mathrm{k}$ and $3 \mathrm{~b}$ show the evolution of the pre-flare activity in AIA $304 \AA$ images. The first brightening in the active region was observed around 16:34 UT. After $\approx 3$ minutes, the brightening becomes more significant (see Figure $3 \mathrm{~b}$ ). Notably, preflare brightening at $\approx 16: 37$ UT agrees with the intensity enhancement seen in the AIA $304 \AA$ time profile of the circular ribbon region shown in Figure $4 \mathrm{~b}$. On 


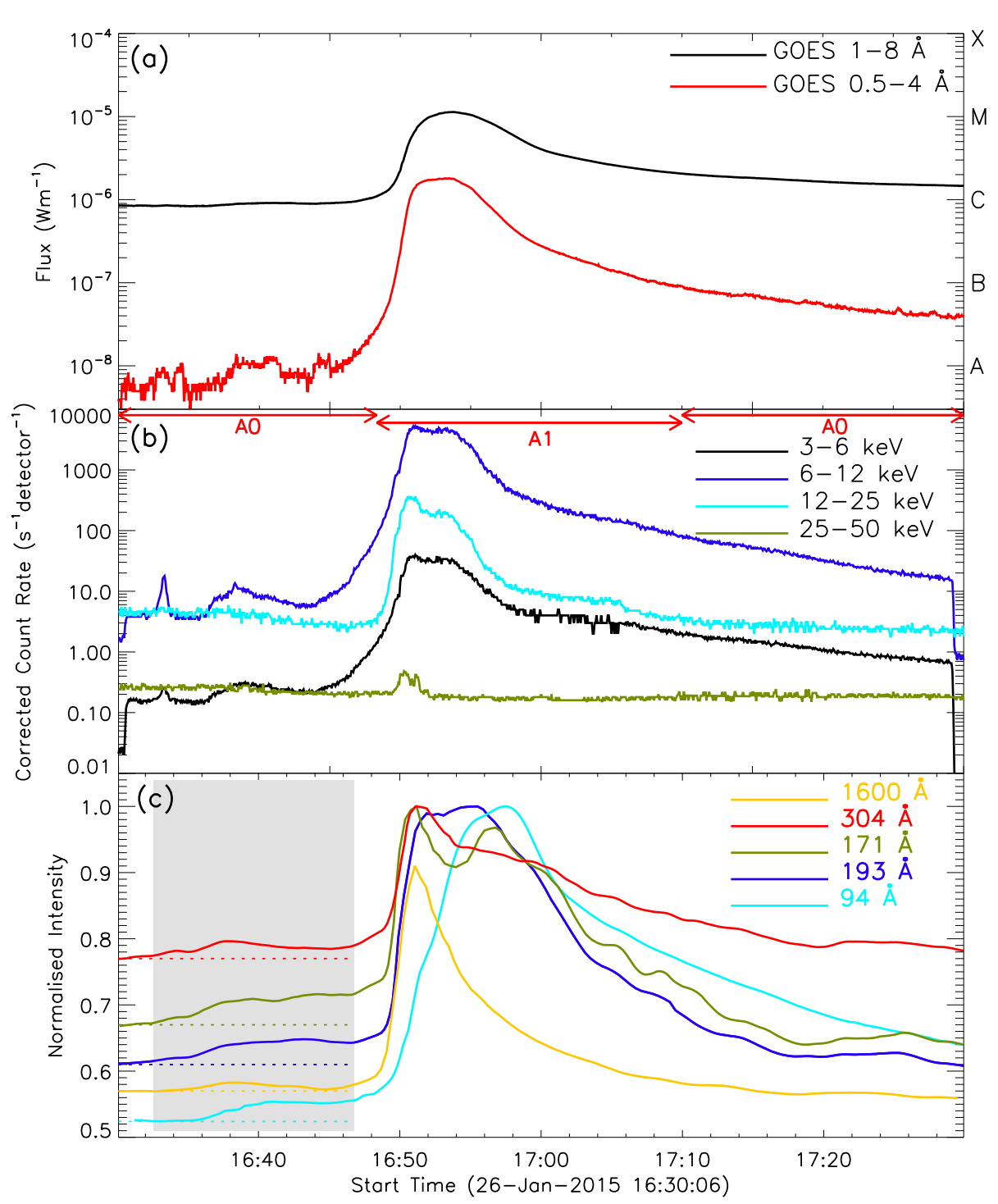

Figure 2. GOES, RHESSI and AIA light curves showing the evolution of the M1.1 class flare. The black and red curves in panel (a) show the GOES X-ray flux in the 1-8 $\AA$ and $0.5-4$ $\AA$ wavelength bands, respectively. Panel (b) shows RHESSI light curves for energy bands of 3-6 keV (black), 6-12 keV (blue), 12-25 keV (sky), and 25-50 keV (green). A0 and A1 denote the RHESSI attenuator states of the RHESSI. Panel (c) shows the light curves of AIA for $94 \AA$ (sky), $193 \AA$ (blue), $171 \AA$ (green), $304 \AA$ (red), and $1600 \AA$ (yellow). The integrated intensity of different channels is normalized by the maximum of corresponding light curve. The grey shaded region corresponds to the pre-flare activity in the same active region. For better visualization, the RHESSI count rates in the 6-12 and 25-50 keV energy bands are scaled by 10 and $1 / 100$, respectively and the normalized intensity corresponding to the AIA UV (1600 $\AA$ ) wavelength is scaled by a factor of 0.9 of its maximum intensity. 
the other hand, the pre-flare brightening in the hot AIA $94 \AA$ filter (temperature $\approx 6 \mathrm{MK})$ or higher energy begins about $\approx 6$ min later $(\approx 16: 40 \mathrm{UT})$. The pre-flare image in AIA $94 \AA$ is displayed in Figure $5 \mathrm{a}$ which confirms the location of the pre-flare brightenings to be co-spatial with the brightest emitting region of the circular ribbon during the peak phase of the flare.

As discussed above, the pre-flare phase was observed in RHESSI measurements up to $12 \mathrm{keV}$ energies. For the whole pre-flare phase, the RHEESI detectors were in the $A 0$ attenuator state, i.e., the imaging was done with the maximum sensitivity limit of the detectors. Therefore, the X-ray images during the pre-flare phase with mild emission are quite reliable. To discuss the spatial evolution of the X-ray sources, we have reconstructed pre-flare images in 3-6 and 6-12 keV energy bands (Figure 6a and 6b). We found single and extended X-ray sources in both energy bands (i.e. 3-6 and 6-12 keV) and the centroid of the sources were almost co-spatial. The location of the X-ray sources were consistent with the EUV brightening. The pre-flare activity suggests that the region where the circular ribbon developed revealed already energy release in the pre-flare phase. This shows the connection between the pre-flare activity and the later main flare reconnection.

\subsection{Development of Circular Ribbon Flare and Remote Brightening}

From the onset of the impulsive phase, some faint circular ribbon flare structure started to appear. As time progressed, the brightening spread in a circular path from $\left[-500^{\prime \prime},-85^{\prime \prime}\right]$ to $\left[-525^{\prime \prime},-65^{\prime \prime}\right]$ in clockwise direction and finally the circular ribbon developed completely during the peak phase of the flare $(\approx 16: 51 \mathrm{UT})$. The development of the circular flare ribbon is presented in Figure 3. Importantly, at higher coronal temperatures (AIA $94 \AA$ images) also, the circular ribbon became visible around 16:51 UT (Figure 5p). However, the intensity of the flaring region in $94 \AA$ channel shows a delayed peak around 16:58 UT as the intensity is dominated by loop brightening over the ribbon structure (see also the accompanying movie in AIA 94 and $304 \AA$ ). To investigate the magnetic field configuration at the flare site, we have overlaid the contours of the HMI line-of-sight magnetic field in AIA $1600 \AA$ image which is displayed in Figure 3. From this figure, we can see that the circular ribbon developed along the circular polarity inversion line.

At $\approx 16: 51$ UT, bright material started to eject along the north-east direction from the brightest south-west part of the circular ribbon and formed ribbon $\mathrm{R}_{2}$. Now looking at the flare morphology around 16:52 UT, we recognize three flare ribbons, $\mathrm{R}_{1}, \mathrm{R}_{2}$, and $\mathrm{R}_{3}$ (shown by arrows in Figure 3 ). Parallel ribbon formation during a circular ribbon flare was also observed by Zhong et al. (2019) from the same active region during 29-30 January 2015. Our flare and the flare studied by Zhong et al. (2019) bear some similarity as well as differences. Zhong et al. (2019) observed that the parallel ribbons converted from the circular ribbon in the west part of the circular ribbon. However, our parallel ribbons are not situated at the location found in the event of Zhong et al. (2019). In our case the conjugate parallel ribbons $\left(\mathrm{R}_{1}\right.$ and $\left.\mathrm{R}_{2}\right)$ form at the eastern part of the circular ribbon. The third ribbon $\left(R_{3}\right)$ is situated at the western part of the circular 


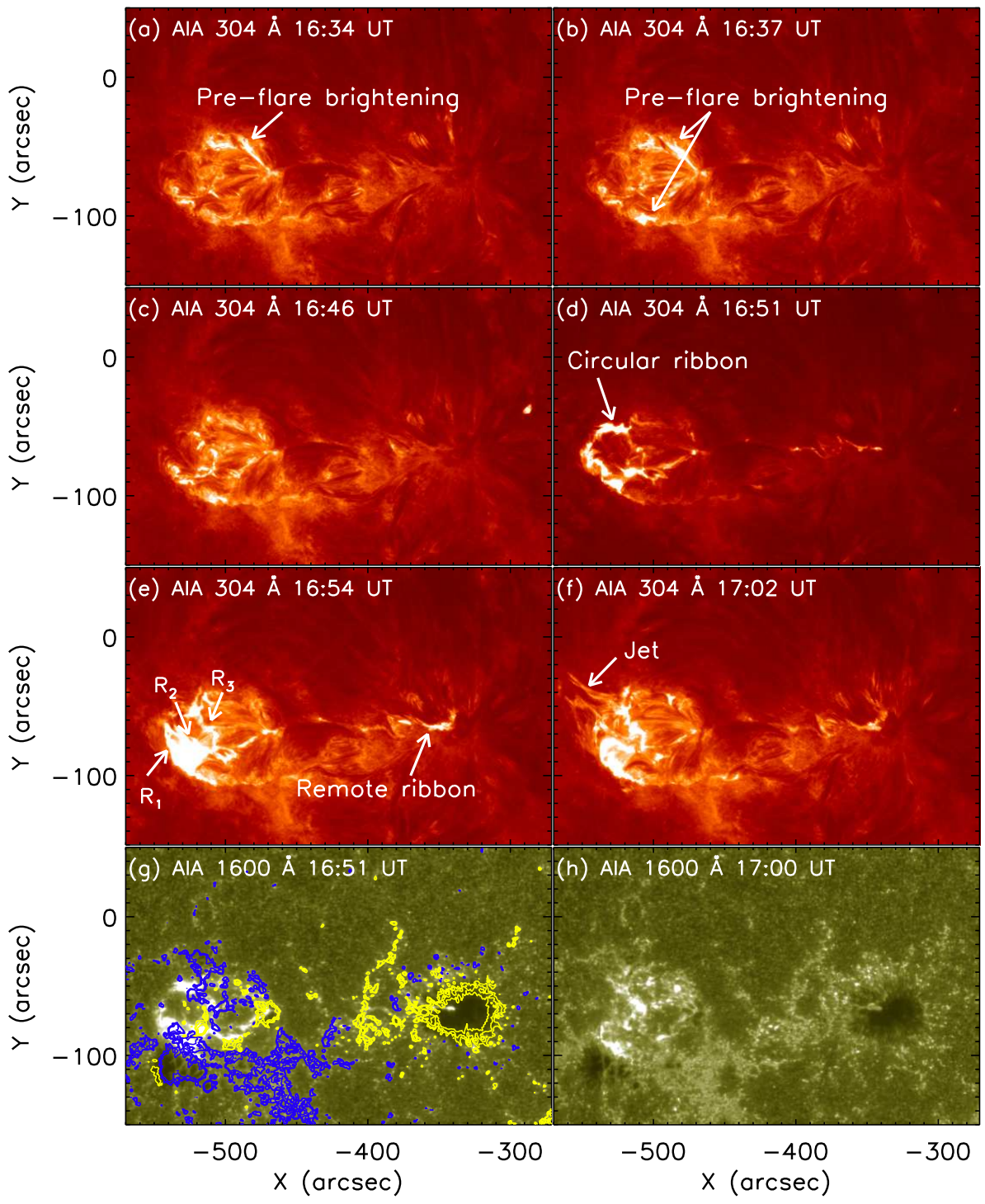

Figure 3. Sequence of AIA $304 \AA$ images showing snapshots during the pre-flare, main flare and post-flare phases: (a) and (b) show the pre-flare images during the times of enhanced emission in GOES, RHESSI, and AIA light curves, (c) corresponds to the impulsive phase of the flare. Image (d) corresponds to the peak time of the event with the circular ribbon indicated by an arrow. The remote brightening, flare ribbons (R1, R2, and R3) and jet structures are shown in (e) and (f), respectively. Panels (g) and (h) show the images in AIA $1600 \AA$ wavelength corresponding to the peak phase of the flare. The yellow and blue contours corresponds to the positive and negative magnetic fields, respectively.

ribbon. We have also noticed the two ribbon $\mathrm{R}_{1}$ and $\mathrm{R}_{2}$ separated as the time 

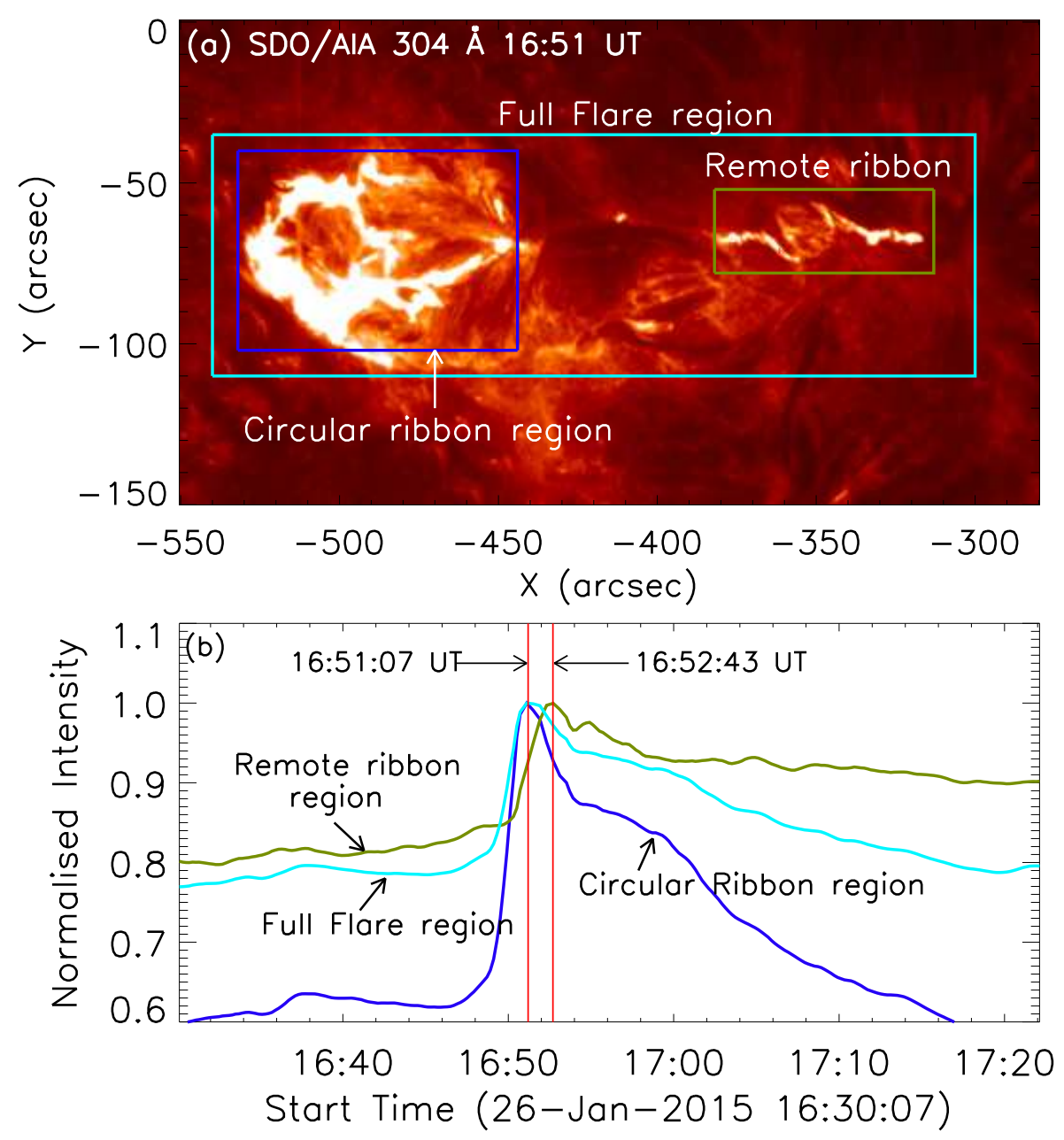

Figure 4. AIA $304 \AA$ image at the flare peak time (16:51 UT) and light curves for the selected regions. Panel (a) shows the flare region where the boxes of cyan, blue, and green colors represent the full flare region, circular ribbon region, and remote brightening region, respectively. The light curves corresponding to these boxes are shown in panel (b). Two vertical red lines in panel (b) show the peak time of the flux for these regions. The observed intensity of each curve is normalized by the corresponding peak intensity.

progresses as commonly observed in the typical two ribbon flares. We could also see clearly the loops connecting these two ribbons.

After $\approx 2$ minutes of the complete development of the circular ribbon a remote brightening appears at the location $\left[-390^{\prime \prime},-70^{\prime \prime}\right], \approx 130^{\prime \prime}$ west from the middle of the circular flaring region. This remote brightening apparently slides $50^{\prime \prime}$ westward after its first appearance and eventually manifested in terms of a well developed remote ribbon. The remote ribbon are displayed in Figures 3e, 4a and 5. From the time profile of region encompassing the remote ribbon, we found that the intensity of remote ribbon peaked $\approx 16: 53 \mathrm{UT}$ (Figure $4 \mathrm{~b}$ ). The 


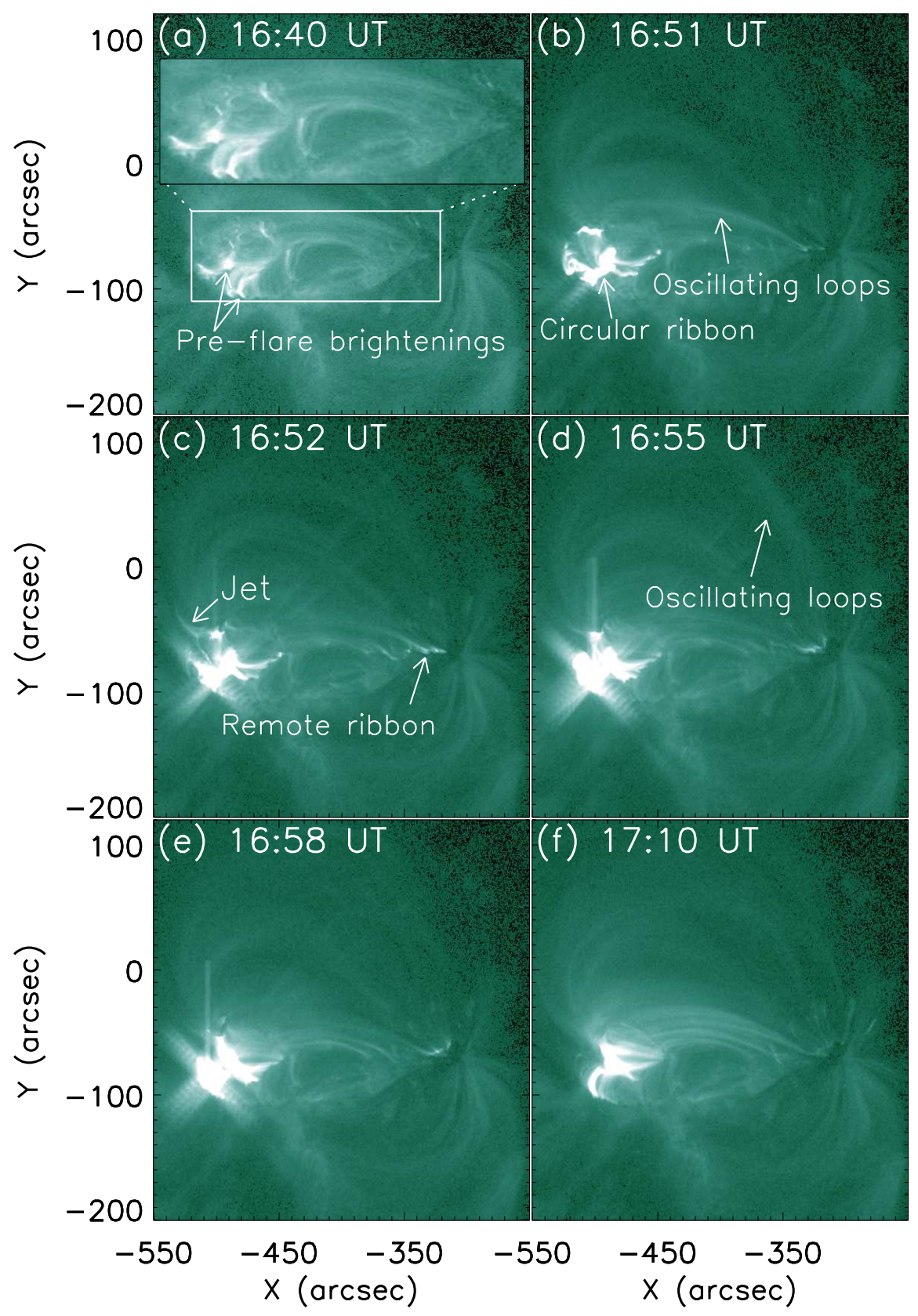

Figure 5. Sequence of AIA $94 \AA$ images. Panel (a) shows pre-flare brightening as marked by arrows. The zoomed view of in the inset of panel (a) shows the pre-flare brightening and the loops connecting the flare region and remote brightening region. In image (b), the circular flare ribbon is shown. We can see the oscillation loops after the reconnection as shown in (b) and (d). The jet and remote brightening associated with the flare are shown in (c). The decay phase of the flare with large-scale hot loops connecting the main and the remote ribbons is shown by (e) and (f). 


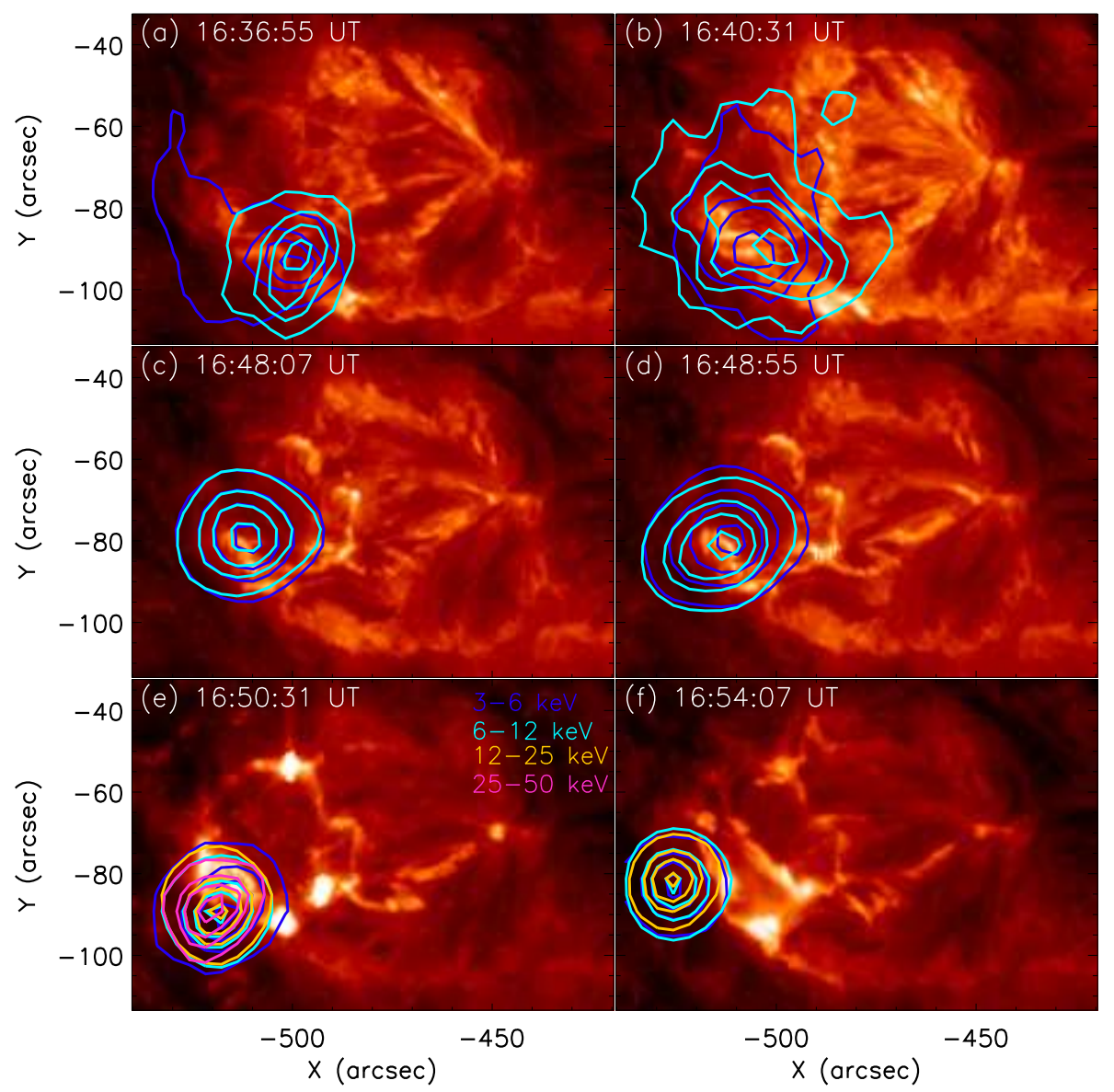

Figure 6. Spatial evolution of RHESSI X-ray sources during the pre-flare phase and main flare. The sources during the pre-flare phase are displayed in panel (a) and (b). From panels (c)-(f), the evolution of X-ray sources during main flare are shown. Here contours of blue, cyan, yellow, and pink color represent the X-ray sources of $3-6 \mathrm{keV}, 6-12 \mathrm{keV}, 12-25 \mathrm{keV}$, and $25-50 \mathrm{keV}$ energy levels, respectively. The images of RHESSI are re-constructed by using CLEAN algorithm. The contour levels are set as $40 \%, 65 \%, 80 \%$, and $95 \%$ of the maximum intensity.

connectivity of the circular ribbon and remote ribbon can be seen in Figure 5 through connecting loops (marked by arrow in Figure 5d). We also observed the oscillation of these loops during the flare.

The spatial evolution of X-ray sources during the main flare phase is presented in Figure 6c-f. Up to the impulsive phase of the flare, we found X-ray sources only at low energies $(3-6 \mathrm{keV}$ and $6-12 \mathrm{keV})$. On the other hand, during the peak phase, X-ray sources at higher energy bands (12-25 keV and $25-50 \mathrm{keV})$ were also detected.

We compare the location of the X-ray sources with AIA/EUV $304 \AA$ images depicted in Figure 6. The co-aligned images shows that the EUV emission and 

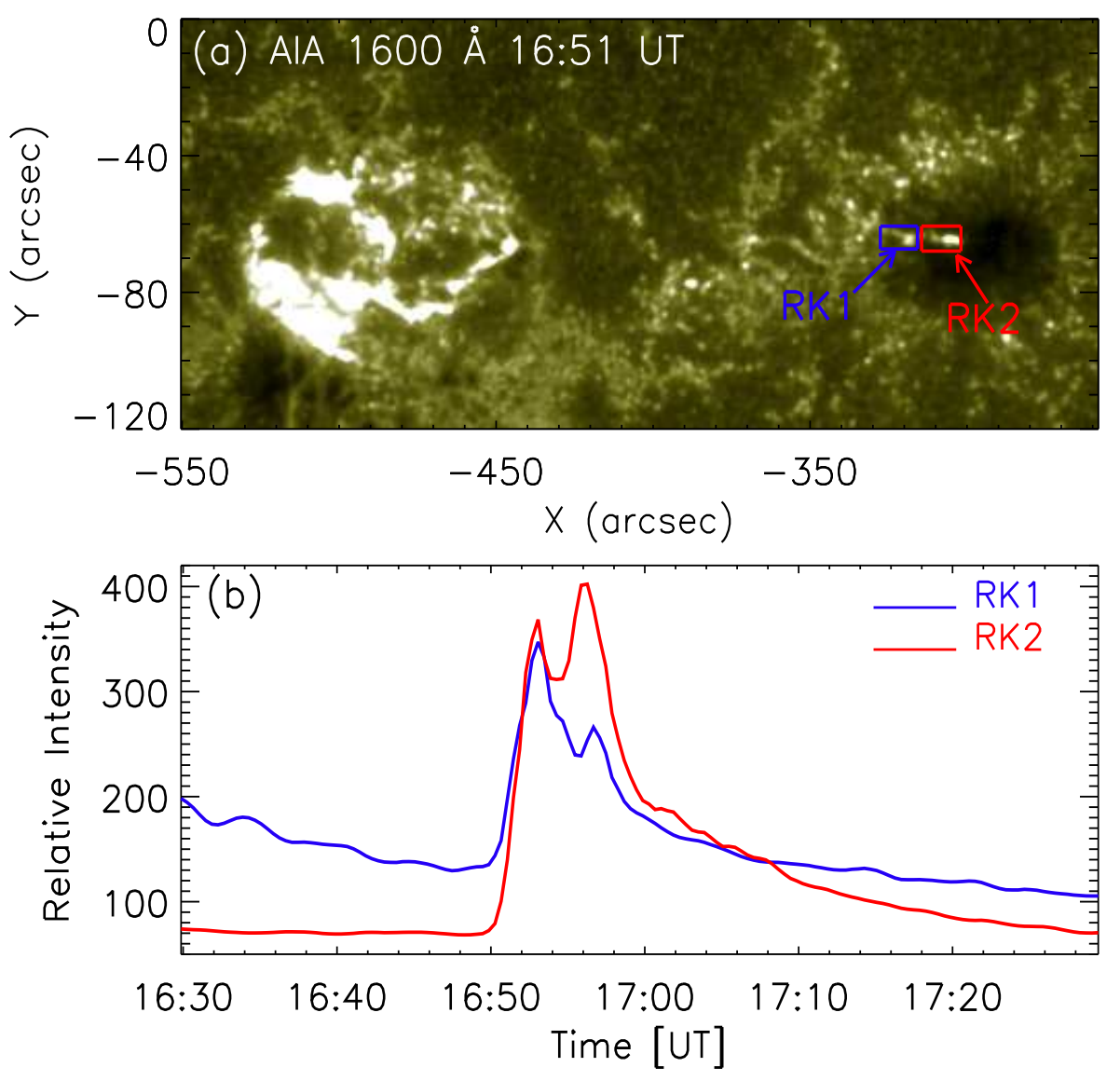

Figure 7. AIA $1600 \AA$ image showing the remote ribbons RK1 and RK2 by blue and red boxes, respectively. The light curve of the two ribbons are displayed in panel (b) showing dual peaks. The light curves has same colour conventions as in panel (a).

X-ray sources are co-spatial up to the early impulsive phase of the flare. During the flare maximum phase $(\approx 16: 50 \mathrm{UT})$, the location of X-ray sources is shifted towards south-east (about $15^{\prime \prime}$ towards east and $4^{\prime \prime}$ towards south). The shift in the locations of EUV and X-ray sources could be due to the fact that the sources at different wavelength originate from different heights of the solar atmosphere. Furthermore, RHESSI imaging analysis reveals that both thermal and non-thermal X-ray sources are co-spatial. The co-spatiality of thermal and non-thermal sources are not uncommon in compact flares (see e.g., Kushwaha et al., 2014). Notably, no distinct foot-point X-ray sources were observed from the locations of flare ribbons $\mathrm{R}_{1}$ and $\mathrm{R}_{2}$. We also tried to reconstruct the X-ray sources in different energy bands at the site of remote ribbon. However, the X-ray sources at this site were absent. In this context, it is noteworthy to report two bright distinct kernels over the remote ribbon in AIA $1600 \AA$ images which are 


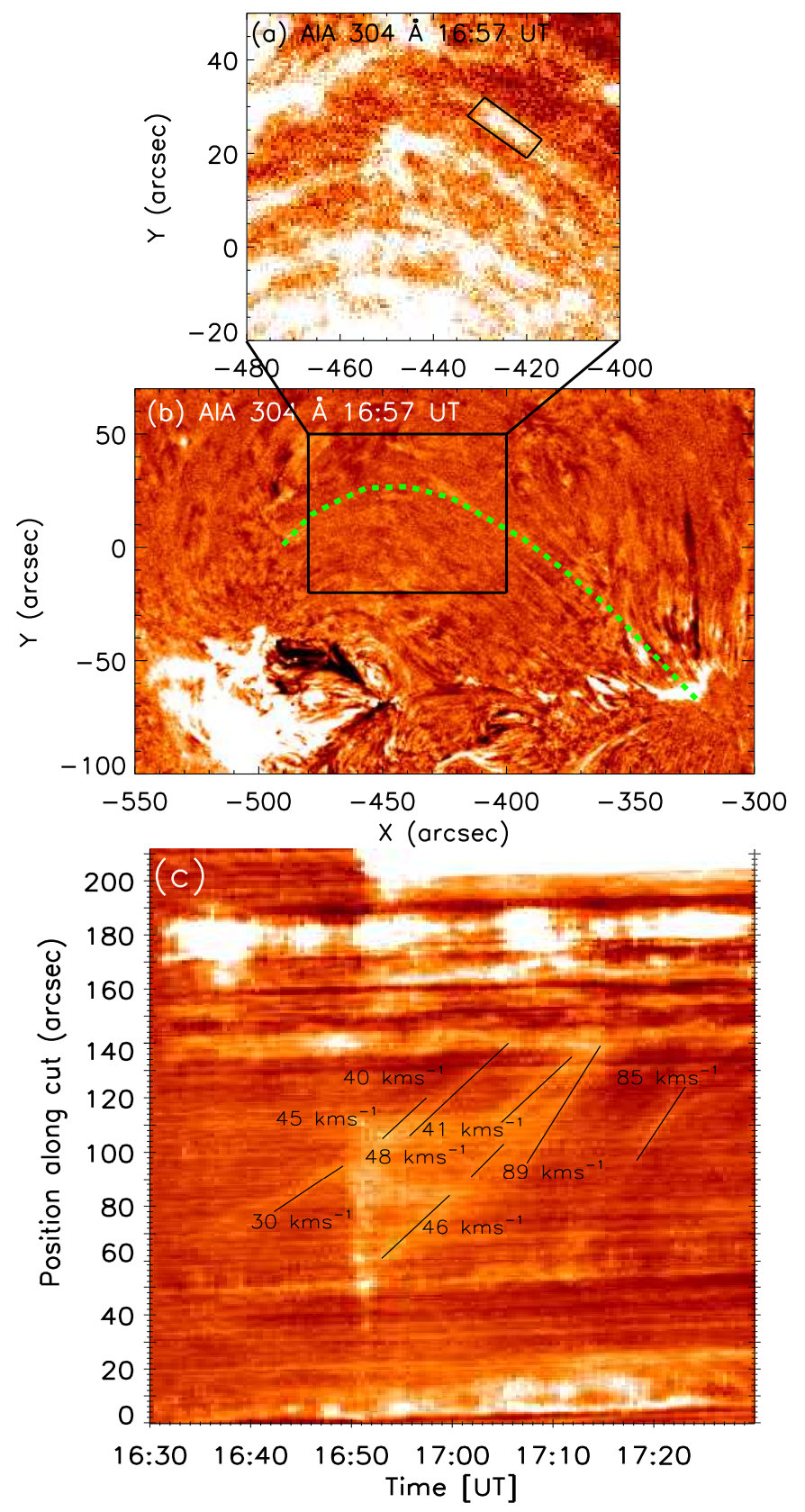

Figure 8. AIA $304 \AA$ images showing the slit (green) taken in the direction of motion of the plasma blob in panel (b). The black rectangle in panel (b) is zoomed in panel (a) which shows the moving plasma blob by a small black box. Panel (c) displayed the time-distance plot corresponding to slice shown in (b). 
presented in Figure $7 \mathrm{a}$ (marked as RK1 and RK2). We present time profiles of intensity of the two kernels in Figure $7 \mathrm{~b}$. The time profiles indicate a dual peak structure with the first one being co-temporal with the overall flare maximum observed by GOES $1-8 \AA$ channel at $\approx 16: 53$ UT. The flare kernels observed in the AIA $1600 \AA$ channel indicate that the source of emission was very compact yet rooted deep up to the upper photospheric levels.

An interesting feature observed in AIA $304 \AA$ images is the flow of plasma at EUV temperatures from the site of circular ribbon to remote ribbon along the overlying field lines. We present some aspects of these plasma flows in Figure 8 . As marked by the black box in the zoomed image of $304 \AA$ (Figure 8 8 ), the plasma flow was observed in the form of moving blobs of plasma. In Figure $8 \mathrm{~b}$, we indicate the trajectory followed by the plasma flow in the $304 \AA$ base difference image by a green dashed line. A time-distance plot along the selected trajectory is shown in Figure 8k. To enhance the bright moving features, the time-distance plot has been constructed by the base difference images where the AIA image at 16:30:19 UT is adopted as the base image. The time-distance diagram captures many instances of moving plasma blobs. However, the flow cannot be observed continuously from the start to the end locations of the chosen trajectory. The speed of plasma blobs ranges from $\approx 30 \mathrm{~km} \mathrm{~s}^{-1}$ to $\approx 90 \mathrm{~km} \mathrm{~s}^{-1}$.

\subsection{Flare Associated Jet Activity}

Around 16:52 UT, while the flare was going through the peak phase, jet activity was observed at the north-east $\left(-500^{\prime \prime},-47^{\prime \prime}\right)$ edge of the circular ribbon. We have indicated the jet activity in the $94 \AA$ and $304 \AA$ images in Figure 9. We notice that in AIA $94 \AA$ wavelength, the jet is visible around 16:52 UT, whereas in $304 \AA$ observations, it can be seen at $\approx 17: 00$ UT. It means that the jet activity was first seen at higher coronal temperatures (i.e. in $94 \AA$ and also in $131 \AA$ wavelength) over the lower temperature emission that originates from transition region and chromospheric layers (i.e. $304 \AA$ wavelength). The estimated speed and projected height of the jet are $\approx 300 \mathrm{kms}^{-1}$ and $\approx 30 \mathrm{Mm}$, respectively, and its life time is $\approx 13 \mathrm{~min}$. The jet activity continued up to 17:10 UT. We can see that along the direction of jet eruption the coronal loops extended up to large coronal heights. The association of jet activity with the circular ribbon flares has been reported in observations and simulations (Pariat, Antiochos, and DeVore, 2010 Wang and Liu, 2012 Li et al., 2018; Zhang, Li, and Huang, 2018).

RHESSI X-ray sources up to $50 \mathrm{keV}$ energies were also observed close to the base of the jet activity. This suggests that the jet material is continuously supplied from the reconnection site. Along with the jet activity, the loops connecting the positive and negative polarity starts to rise from the following polarity site, where the circular ribbon was formed. Contrary to this, the other end of the loop system was fixed. Through these connecting loops, the accelerated non-thermal particles injected from the coronal reconnection region at the following polarity can travel towards the distant foot-points of the leading polarity causing remote brightenings at that location. The remote brightening appeared at several places in the eastern part of the leading polarity sunspot. 


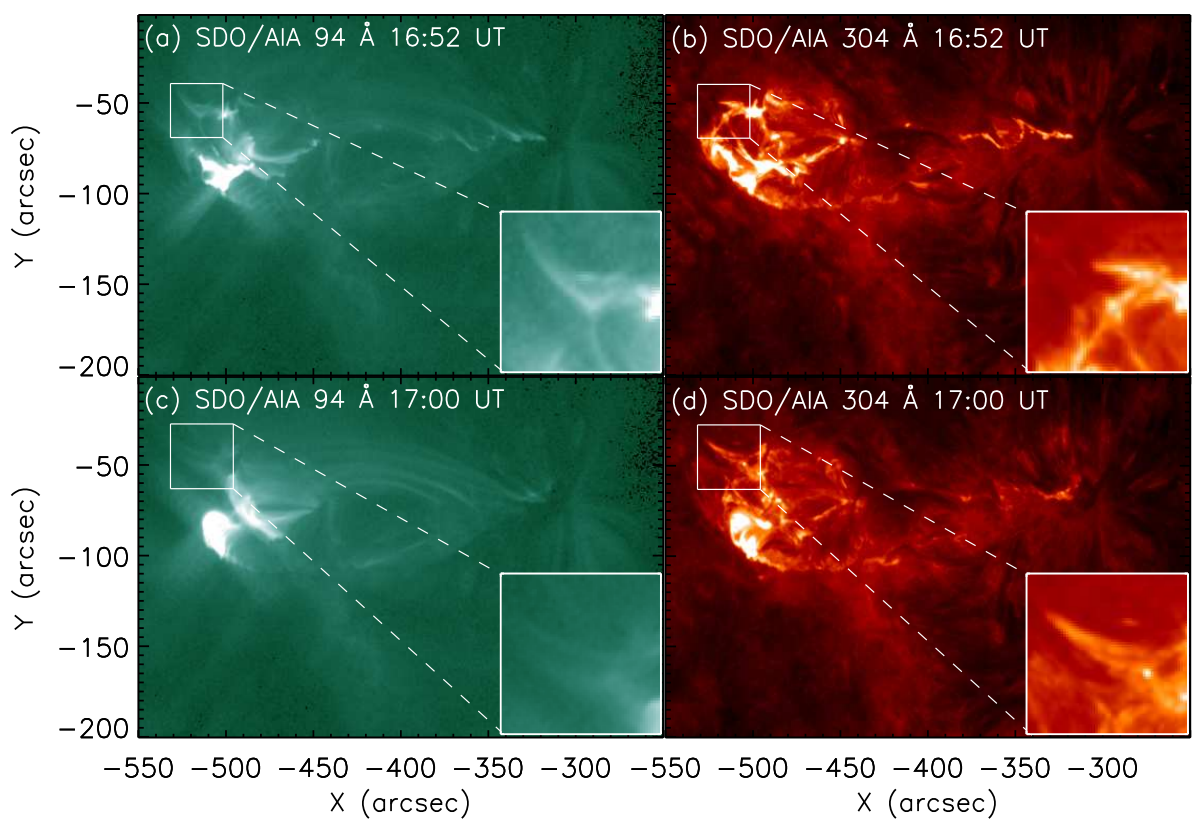

Figure 9. SDO/AIA $94 \AA$ and $304 \AA$ images showing the jet activity associated with the flare region. The inset shows an enlarged view of the jet.

\subsection{Magnetic Field Evolution and Topology of the Active Region}

\subsubsection{Photospheric Structure}

The occurrence of flare and associated phenomena are closely related to the active region's magnetic field configuration. The evolution of the magnetic field is depicted in Figure 10. When the active region appeared on the disk, it exhibited mixed magnetic polarities at both the circular ribbon flare site and the remote ribbon site. The flaring region (shown with the dashed box in Figure 10p) shows the continuous emergence of photospheric magnetic flux of both polarities for a long period prior to the flare on 26 January 2015. A few of the dispersed patches of the magnetic field from this region are of special interest, we mark them as $\mathrm{P}_{1}$, $\mathrm{P}_{2}, \mathrm{~N}_{1}, \mathrm{~N}_{2}, \mathrm{~N}_{3}$, and $\mathrm{N}_{4}$ (shown in Figure 10d). As time progressed, a part of $\mathrm{P}_{1}$ displayed a slow westward motion and a negative polarity region emerged behind the slowly moving $\mathrm{P}_{1}$, which is indicated as $\mathrm{N}_{5}$ in Figure 10 . Simultaneously, we also observed migration of a positive polarity patch (indicated as $\mathrm{P}_{3}$ in Figure 10: towards $\mathrm{N}_{1}$. The positive polarity $\mathrm{P}_{1}$ further moved towards $\mathrm{P}_{2}$ and finally $\mathrm{P}_{1}$ and $\mathrm{P}_{2}$ merged around 16:34 UT. Now, we recognize that at circular ribbon site, the negative polarity patches $\left(\mathrm{N}_{1}-\mathrm{N}_{5}\right)$ surrounded the central positive polarity making the entire arrangement similar to an anemone configuration. This anemone type photospheric structure was co-spatial with the circular ribbon of the flare, which is shown by red dashed circle in Figure $10 \mathrm{~g}$. 

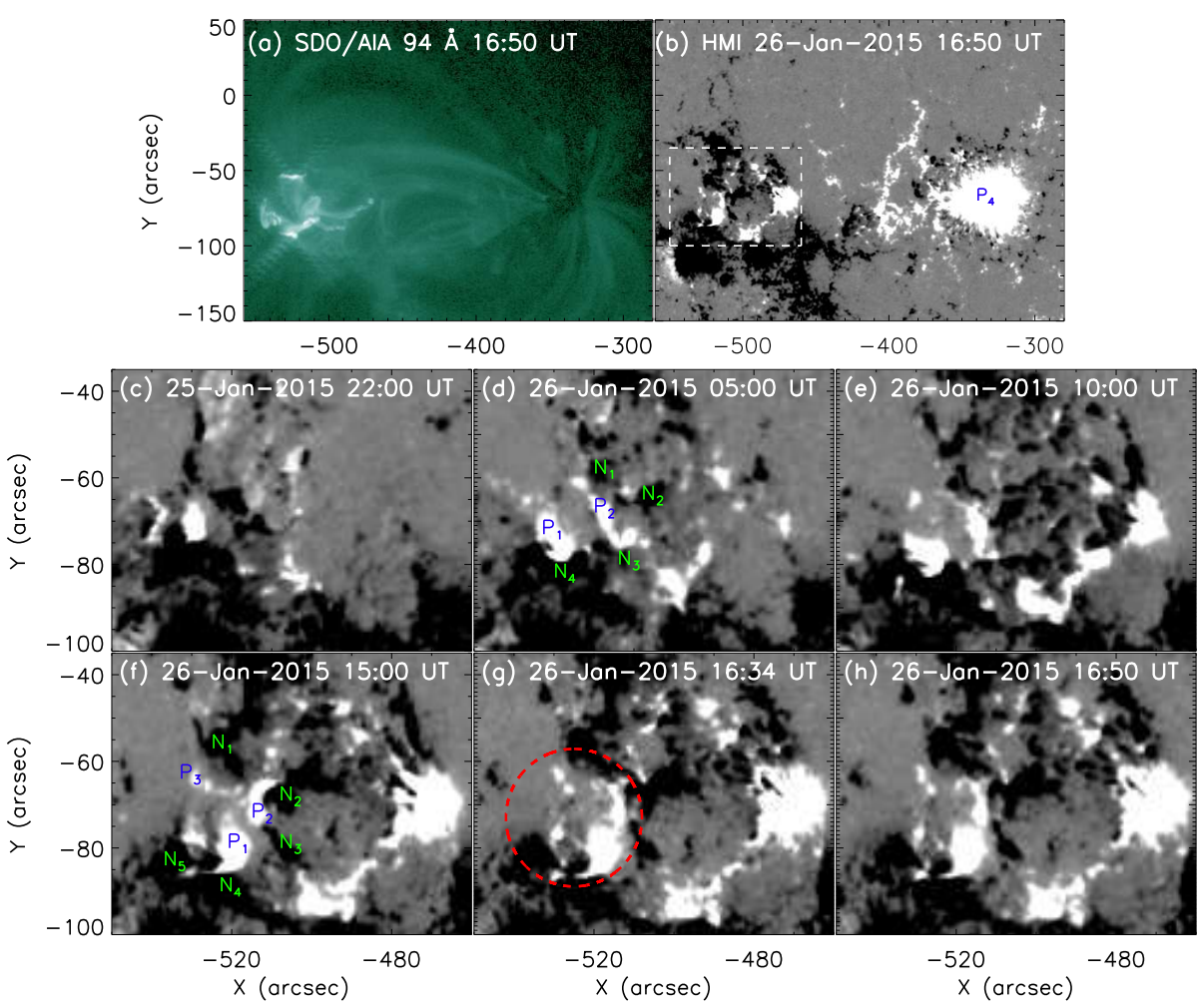

Figure 10. Evolution of the magnetic field in the flare region. Panel (a) shows an AIA $94 \AA$ image at 16:50 UT showing the circular ribbon of the flare. Panel (b) shows an HMI magnetogram of the flare region by dashed box and the remote brightening region at $\mathrm{P}_{4}$. The evolution of the magnetic field for the flaring region with enlarged view of this box is displayed in panels $(\mathrm{c})-(\mathrm{h})$. Red dashed circle in panel $(\mathrm{g})$ indicate the location of the circular flare ribbon.

We also observe continuous emergence and cancellation of the flux at remote brightening site, where positive polarity is more magnetically dominated. We name this positive polarity as $\mathrm{P}_{4}$ (shown in Figure $10 \mathrm{p}$ ).

\subsubsection{Nonlinear Force Free Field (NLFFF) Extrapolation}

To investigate the coronal magnetic configuration associated with the active region, we have performed a non-linear force free field (NLFFF) extrapolation using the advanced version of an optimization code developed by Wiegelmann et al. (2007) and Wiegelmann and Inhester (2010). In Figure 11, we have displayed a few of the modelled field lines representing the coronal connectivities prior to the flare to show the coronal magnetic field configuration of the active region from different viewing angles. The NLFFF extrapolation result suggests the presence of a fan-spine configuration involving a coronal null-point in the active region. Notably, the fan lines (shown by yellow colour in Figure 11 a $-\mathrm{d}$ ) originated from the mixed polarity trailing region which was associated with 

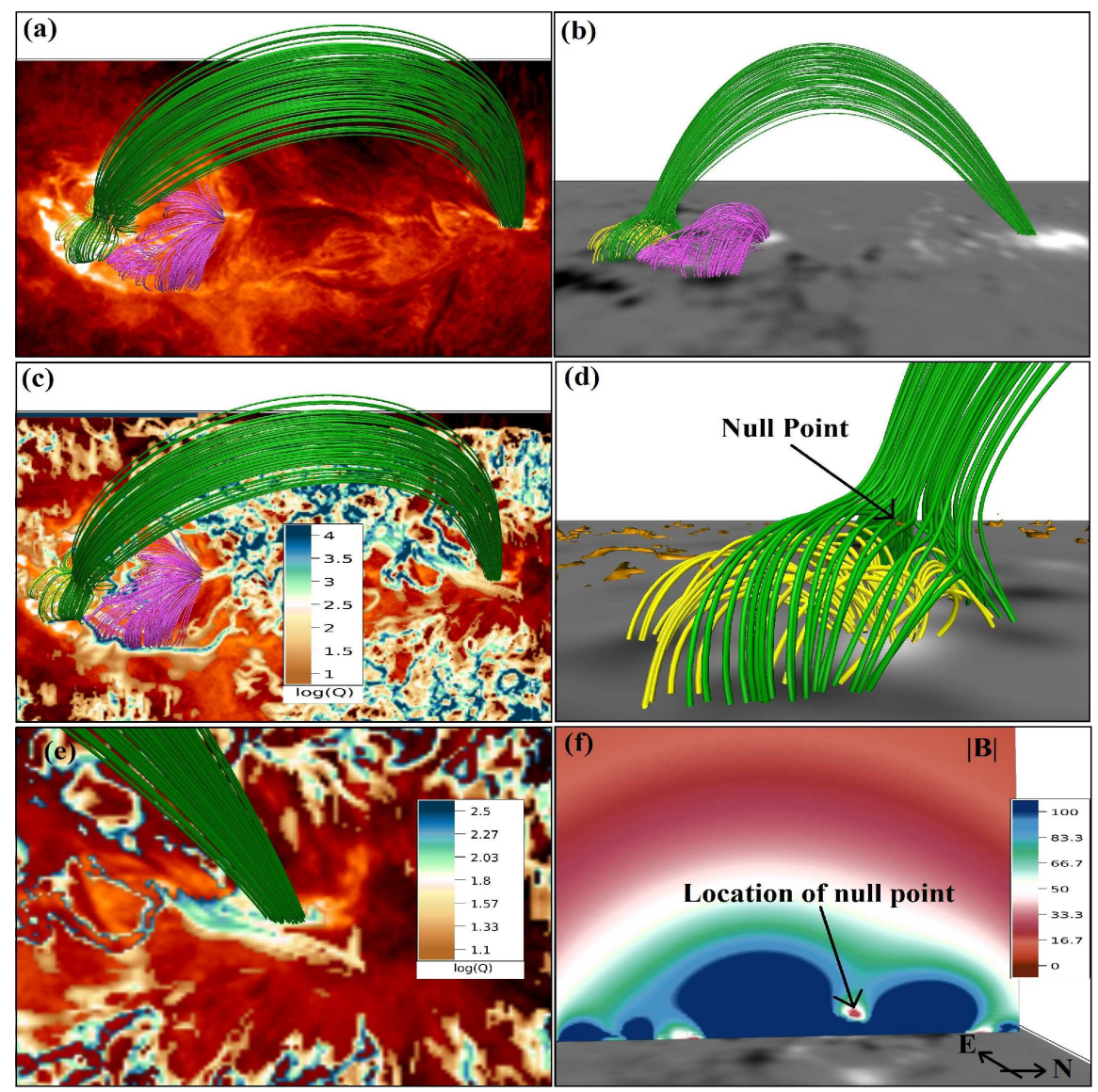

Figure 11. Panel (a): Non linear force free field (NLFFF) lines associated with the active region NOAA 12268 during the pre flare phase. The yellow and green lines form a typical fan-spine configuration. The circular ribbon region involved the fan-spine configuration along with the pink lines which are a set of low coronal closed magnetic loops. For comparison we have plotted an AIA 304 image showing the circular ribbons at the base of the extrapolation volume. Panel (b): NLFFF lines of the overall active region from the different angle. In panel (c) we plot the degree of squashing factor $(Q)$ on the background AIA $304 \AA$. Panel (d): The fan configuration along with a part of the spine lines showing the approximate location of the null-point (shown by the orange iso-surface). In panel (e) we plot only the remote ribbon region along with the associated $\log (Q)$ values between [1.1, 2.5]. Panel (f): A vertical surface passing through the null-point showing the variation of magnetic field strength $(|\mathrm{B}|)$. Note that, the surface in panel (d) is along $\mathrm{Y}-\mathrm{Z}$ orientation and we have indicated the north and east directions in the same panel for convenience.

the circular ribbon brightening (highlighted by the dashed circle in Figure $10 \mathrm{~g}$ ). This mixed polarity region region was also connected with the leading positive polarity sunspot (indicated by $\mathrm{P}_{4}$ in Figure $10 \mathrm{p}$ ), by a set of large closed lines (spine lines). 
As described in sub-section 3.3 and displayed in AIA $94 \AA$ image of Figure 5 . we have found that the main circular ribbon flare and the jet site is connected to the remote flare site through high-lying coronal loops. The jet material ejects along these high-lying coronal loops. We have modelled these loops using the NLFFF extrapolation and found two type of field lines (shown in Figure 11): first are yellow low-lying field lines (fan-structure) and the second are green highlying field lines (spine-structure). Usually the jets are observed at the location of open magnetic field lines and newly emerged magnetic flux, which formed small field lines (see e.g., Chandra et al., 2017, Joshi et al., 2017 and references cited therein). However, in our case we found that a set of large-overlying field line originate from the jet location which closes at larger distances. Thus, it is likely that the coronal jet resulted as the low-lying bipolar loops within the circular flare area interacted with the large, apparently uni-polar field lines essentially forming leg of a high-lying coronal loop system. Therefore, our present case evidenced that the observed jet is due to the reconnection between the low-lying and the high-lying magnetic field lines. Such a scenario was also reported in previous observations (Bentley et al., 2000, Uddin et al., 2012). In such type of confined magnetic configuration, the material ejected from the jet and associated accelerated particles are expected to largely remain confined in closed high-lying loops.

In order to find the photospheric regions with high connectivity gradient, we calculated the degree of squashing factor $(Q)$ by employing the code developed by Liu et al. (2016) based on the NLFFF extrapolation results. From Figure 11., we readily find that the circular ribbon region was characterized by the highest $Q$ values in the active region $(\log (Q)>3.5)$. Further it is also noticeable that, the regions associated with the remote ribbon had moderately higher values of $Q$ compared to the ribbon-less regions. From Figure 11, we find that the remote brightening region was associated with $Q$ values between $\approx 30$ and 200 $(1.5>\log (Q)>2.3)$.

\section{Discussion and Conclusion}

In this article, we investigate an M1.1 class solar flare of 26 January 2015. An important finding of this study lies in the complex morphological evolution of flare ribbon in anemone-type magnetic field configuration within active region NOAA 12268, where this confined M1.1 class flare occurred. The flare rises with the development of the circular ribbon which eventually proceeds with three parallel ribbon structures within the same region. The main results of our study are summarized as below:

i) We have observed the pre-flare activity in the active region that spans for $\approx 15 \mathrm{~min}$ in EUV/UV as well as in X-ray energy bands. This pre-flare activity could provide an important role for the triggering of main circular ribbon flare.

ii) The evolution of loops visible in AIA $193 \AA$ and $94 \AA$ reveals following connectivity: We observed the loops originating from the circular ribbon 
region connect and converge to the distant positive polarity sunspot region such that the overall structure resembles with fan-spine configuration. In the decay phase of flare, where two ribbons appeared at the south-east site, we observed the post-flare loops as visible in a typical two ribbon flares. NLFFF extrapolation of the active region evidenced the fan-spine magnetic topology and the presence of null-point in the active region.

iii) The remote flare ribbon exhibited fine structured kernels. An important characteristic of remote ribbon is the progression of brightness from east to west direction. Further, photospheric region corresponding to the remote ribbon indicates relatively higher values of degree of squashing factor $(Q)$ that lie in the range of $\approx 30$ and 200 , providing evidence of slipping reconnection.

We have observed the pre-flare activity in the active region prior to $\approx 15 \mathrm{~min}$ of the main flare. The pre-flare activity in solar flares were also reported in the past literature (Veronig et al., 2002, Kim et al., 2007, Chandra et al., 2009, Joshi et al., 2011; Awasthi et al., 2014 Wang et al., 2017 Hernandez-Perez et al., 2019. Mitra and Joshi, 2019, Mitra, Joshi, and Prasad, 2020). In our study, the cospatiality of the pre-flare and main flare emitting regions in X-ray and EUV/UV provides evidence that pre-flare activity has likely contributed toward triggering of the main flare. In this context, it is noteworthy that in terms of morphology of hot plasma structures the pre-flare and the main flare present similarities. The regions of pre-flare brightenings continued to be visible during the main flare, albeit with variation in intensity ( $c f$. Figure $3 \mathrm{p}$ and $3 \mathrm{~d}$ ).

To find a plausible interpretation of the observed pre-flare activity, we examine the corresponding region in the photospheric magnetograms during the period of pre-flare activity (Figure $10 \mathrm{~g}$ ). By this time, the photospheric magnetic field configuration of the flaring region had evolved into an "anemone" type configuration (see the region delineated by dashed circle in Figure $10 \mathrm{~g}$ ). The NLFFF extrapolation suggests a fan-spine configuration involving a coronal nullpoint with outer fan-lines being anchored at the periphery of the photospheric anemone structure (Figure $11 \mathrm{a}-\mathrm{d}$ ). These observations indicate that the pre-flare brightening, occurring within the periphery of later developed circular ribbon, is suggestive of the ongoing null-point reconnection which is quite subtle at this time with no detectable emission at the location of remote ribbons. We further emphasize that the pre-flare magnetic configuration, as discussed above, has evolved as a result of continuous flux emergence and cancellation (see Figure 10 and section 3.4.1). In such regions, the pre-flare brightenings are also attributed to low-atmosphere magnetic reconnection (e.g. Moon et al., 2004, Wang et al., 2017). Thus our observations suggest the contribution from both the processes described above that eventually led to a mild yet continuous energy release $\approx 15$ min prior to the main circular ribbon flare. Green et al., 2018 presents a review of the predictability of solar eruptions. They have discussed that the numerical simulations and observations (see their table 1) provide evidences that the pre-flare configuration play an important role for the major flare activity. The pre-flare activity lower the magnetic tension in the active region and as a result of this the active region produced main flare. 
The most noticeable characteristic of the M1.1 flare is the development of a circular ribbon structure and brightening at a distant location which eventually progresses into the remote ribbon (Figure 4). The circular ribbon is visible in almost all AIA EUV/UV filters. The circular ribbon structures and associated remote brightening can be explained by magnetic reconnection at a coronal nullpoint located in the fan-spine configuration (Masson et al., 2009; Reid et al., 2012 Wang and Liu, 2012 Pontin, Galsgaard, and Démoulin, 2016 Hao et al., 2017. Hernandez-Perez et al., 2017; Li et al., 2018; Zhang, Li, and Huang, 2018). The topology of the flaring region, inferred from the NLFFF extrapolation, readily supports this scenario (Figure $11 \mathrm{a}-\mathrm{c}$ ) where we find a good match between observed and modelled structures. These observed structures include the circular ribbon, remote ribbon, and the overlying loop system connecting the circular and remote ribbons. We observed a time delay of $\approx 2 \mathrm{~min}$ in the appearance of remote brightenings with respect to the circular ribbon appearance (Figure 4). The time delay in the evolution of the circular and remote ribbons has been well documented in previous studies (e.g. Wang and Liu, 2012, Li et al., 2018).

During the main phase of the M-class flare, we notice moving blobs of plasma that apparently follow the overlying coronal loops connecting the circular and remote ribbons. The time-distance diagram based on base difference images (Figure 8) clearly indicates these plasma flows but they occur within a limited range of distances only. Hernandez-Perez et al. (2017) reported plasma flows toward the formation site of remote ribbons from the location of the circular ribbon in the flare of 29 January 2015, which occurred in the same active region. However, unlike our case, the plasma flows in their event occurred during the pre-flare phase (as early as $15 \mathrm{~min}$ before the onset) and the continuous flow patterns were visible between the two distant ribbon locations. Hernandez-Perez et al. (2017), therefore, concluded that the remote brightenings were caused by the dissipation of kinetic energy of the plasma flows. In our observations, the moving plasma blobs appear as temporary structures that fade away much before reaching to the location of the remote ribbon. Therefore, it is more likely that the remote ribbon in our case is primarily formed by the interaction of nonthermal particles, flowing along the overlying coronal loop system connecting the location of circular ribbon to its remote footpoint, which is well observed in AIA $94 \AA$ images (see the movie accompanying Figure 5). Our interpretation is consistent with the mechanism of typical circular ribbon flares proposed in some of the earlier studies (e.g. Tang and Moore, 1982, Nakajima et al., 1985, Wang and Liu, 2012). The streams of accelerated particles will eventually be collisionally stopped by the denser layers of the lower solar atmosphere creating the fine-structured kernels of the remote ribbon.

A careful examination of the remote ribbon suggests the spatial progression of brightening from east to west direction. In this context, from Figure 11, we note that the remote brightening region is associated with high degree of squashing factor $(Q)$. The calculated value of this $Q$ is found in between 30 and 200. From such high values of $Q$, the motion of the remote brightening can be interpreted in terms of slipping reconnection in this region (Chandra et al., 2011; Aulanier, Janvier, and Schmieder, 2012, Dudík et al., 2014, Li and Zhang, 2014, 2015. Janvier, Aulanier, and Démoulin, 2015. Dudík et al., 2016. Zheng, Chen, and 
Wang, 2016). In fan-spine topology, the slipping magnetic reconnection in QSLs and null-point reconnection can occur simultaneously (Masson et al., 2009 Reid et al., 2012, Pontin, Galsgaard, and Démoulin, 2016).

Before the main flare, significant amount of the positive magnetic flux emerged in the active region (Figure 10). This positive polarity flux emergence got distributed within the pre-existing patches of negative polarity in such a way that an overall anemone-type magnetic configuration appeared in the photosphere (Asai et al., 2008). This kind of magnetic flux distribution in the photosphere is favorable for the fan-spine structure in the corona.

The NLFFF extrapolation reveals the presence of 3D null at a relatively lower height i.e. $10 \pm 1 \mathrm{Mm}$ (Figure 11:). The appearance of flare brightening in a quasicircular pattern right from pre-flare phase, therefore, suggests energy release due to ongoing 3D reconnection which get enhanced during flare's main phase. The formation of parallel ribbons within the periphery of the circular ribbon suggests onset of standard flare reconnection in the later phase of M1.1 flare. The formation of parallel ribbon in a confined flare is rather surprising and may point toward the small scale flux rope eruption (invisible in direct observation and extrapolation) or interaction between different flux systems within the fandome. These complex observations put constraints on contemporary models of solar flare. The observations of jet activity in the main flare site also support the idea that the magnetic reconnection was initiated at a coronal null-point (Pariat, Antiochos, and DeVore, 2010, Liu et al., 2011; Zeng et al., 2016).

Acknowledgments We would like to thank the referee for valuable comments and suggestions. SDO is a mission for NASA's Living With a Star (LWS) Program. SDO data are courtesy of the NASA/SDO and HMI science team. RHESSI is a NASA Small Explorer Mission. GOES is a joint effort of NASA and the National Oceanic and Atmospheric Administration (NOAA). $\mathrm{PD}, \mathrm{RC}$ and BJ acknowledges the support from the DST/SERB project. PD also acknowledges the support from the CSIR, New Delhi. This work is supported by the Indo-Austrian joint research project no. INT/AUSTRIA/BMWF/ P-05/2017 and OeAD project no. IN 03/2017. A.M.V. acknowledges the Austrian Science Fund ( FWF) : P25383-N27. RJ thanks to the Department of Science and Technology (DST), New Delhi, India for an INSPIRE fellowship.

Disclosure of Potential Conflicts of Interest The authors declare that they have no conflicts of interest.

\section{References}

Asai, A., Shibata, K., Hara, H., Nitta, N.V.: 2008, Characteristics of Anemone Active Regions Appearing in Coronal Holes Observed with the Yohkoh Soft X-Ray Telescope. Astrophys. J. 673, 1188. DOI ADS

Aulanier, G., Janvier, M., Schmieder, B.: 2012, The standard flare model in three dimensions. I. Strong-to-weak shear transition in post-flare loops. Astron. Astrophys. 543, A110. DOI ADS

Awasthi, A.K., Jain, R., Gadhiya, P.D., Aschwanden, M.J., Uddin, W., Srivastava, A.K., Chandra, R., Gopalswamy, N., Nitta, N.V., Yashiro, S., Manoharan, P.K., Choudhary, D.P., Joshi, N.C., Dwivedi, V.C., Mahalakshmi, K.: 2014, Multiwavelength diagnostics of the precursor and main phases of an M1.8 flare on 2011 April 22. Mon. Not. Roy. Astron. Soc. 437, 2249. DOI ADS 
Bentley, R.D., Klein, K.-L., van Driel-Gesztelyi, L., Démoulin, P., Trottet, G., Tassetto, P., Marty, G.: 2000, Magnetic Activity Associated With Radio Noise Storms. Solar Phys. 193, 227. DOI ADS

Carmichael, H.: 1964, A Process for Flares. NASA Special Publication 50, 451. ADS

Chandra, R., Schmieder, B., Aulanier, G., Malherbe, J.M.: 2009, Evidence of Magnetic Helicity in Emerging Flux and Associated Flare. Solar Phys. 258, 53. DOI. ADS

Chandra, R., Schmieder, B., Mandrini, C.H., Démoulin, P., Pariat, E., Török, T., Uddin, W.: 2011, Homologous Flares and Magnetic Field Topology in Active Region NOAA 10501 on 20 November 2003. Solar Phys. 269, 83. DOI ADS.

Chandra, R., Filippov, B., Joshi, R., Schmieder, B.: 2017, Two-Step Filament Eruption During 14 - 15 March 2015. Solar Phys. 292, 81. DOI ADS

Dudík, J., Janvier, M., Aulanier, G., Del Zanna, G., Karlický, M., Mason, H.E., Schmieder, B.: 2014, Slipping Magnetic Reconnection during an X-class Solar Flare Observed by SDO/AIA. Astrophys. J. 784, 144. DOI ADS

Dudík, J., Polito, V., Janvier, M., Mulay, S.M., Karlický, M., Aulanier, G., Del Zanna, G., Dzifčáková, E., Mason, H.E., Schmieder, B.: 2016, Slipping Magnetic Reconnection, Chromospheric Evaporation, Implosion, and Precursors in the 2014 September 10 X1.6-Class Solar Flare. Astrophys. J. 823(1), 41. DOI ADS

Fletcher, L., Dennis, B.R., Hudson, H.S., Krucker, S., Phillips, K., Veronig, A., Battaglia, M., Bone, L., Caspi, A., Chen, Q., Gallagher, P., Grigis, P.T., Ji, H., Liu, W., Milligan, R.O., Temmer, M.: 2011, An Observational Overview of Solar Flares. Space Sci. Rev. 159, 19. DOI. ADS

Green, L.M., Török, T., Vršnak, B., Manchester, W., Veronig, A.: 2018, The Origin, Early Evolution and Predictability of Solar Eruptions. Space Sci. Rev. 214(1), 46. DOI ADS

Hao, Q., Yang, K., Cheng, X., Guo, Y., Fang, C., Ding, M.D., Chen, P.F., Li, Z.: 2017, A circular white-light flare with impulsive and gradual white-light kernels. Nature Communications 8, 2202. DOI ADS

Harvey, J.W., Bolding, J., Clark, R., Hauth, D., Hill, F., Kroll, R., Luis, G., Mills, N., Purdy, T., Henney, C., Holland, D., Winter, J.: 2011, Full-disk Solar H-alpha Images From GONG. In: AAS/Solar Physics Division Abstracts \#42, Bulletin of the American Astronomical Society 43, 17.45. ADS

Hernandez-Perez, A., Thalmann, J.K., Veronig, A.M., Su, Y., Gömöry, P., Dickson, E.C.: 2017, Generation Mechanisms of Quasi-parallel and Quasi-circular Flare Ribbons in a Confined Flare. Astrophys. J. 847(2), 124. DOI ADS

Hernandez-Perez, A., Su, Y., Veronig, A.M., Thalmann, J., Gömöry, P., Joshi, B.: 2019, Preeruption Processes: Heating, Particle Acceleration, and the Formation of a Hot Channel before the 2012 October 20 M9.0 Limb Flare. Astrophys. J. 874(2), 122. DOI. ADS

Hirayama, T.: 1974, Theoretical Model of Flares and Prominences. I: Evaporating Flare Model. Solar Phys. 34, 323. DOI ADS

Hong, J., Jiang, Y., Yang, J., Li, H., Xu, Z.: 2017, Minifilament Eruption as the Source of a Blowout Jet, C-class Flare, and Type-III Radio Burst. Astrophys. J. 835, 35. DOI ADS

Janvier, M., Aulanier, G., Démoulin, P.: 2015, From Coronal Observations to MHD Simulations, the Building Blocks for 3D Models of Solar Flares (Invited Review). Solar Phys. 290, 3425. DOI ADS

Jiang, C., Feng, X.: 2013, Extrapolation of the Solar Coronal Magnetic Field from SDO/HMI Magnetogram by a CESE-MHD-NLFFF Code. Astrophys. J. 769, 144. DOI. ADS

Joshi, B., Veronig, A.M., Lee, J., Bong, S.-C., Tiwari, S.K., Cho, K.-S.: 2011, Pre-flare Activity and Magnetic Reconnection during the Evolutionary Stages of Energy Release in a Solar Eruptive Flare. Astrophys. J. 743, 195. DOI ADS.

Joshi, R., Schmieder, B., Chandra, R., Aulanier, G., Zuccarello, F.P., Uddin, W.: 2017, Slippage of Jets Explained by the Magnetic Topology of NOAA Active Region 12035. Solar Phys. 292, 152. DOI ADS

Kim, S., Moon, Y.-J., Kim, K.-H., Kim, Y.-H., Sakurai, T., Chae, J., Kim, K.-S.: 2007, TwoStep Reconnections in a C3.3 Flare and Its Preflare Activity Observed by Hinode XRT. Pub. Astron. Soc. Japan 59, S831. DOI ADS.

Kopp, R.A., Pneuman, G.W.: 1976, Magnetic reconnection in the corona and the loop prominence phenomenon. Solar Phys. 50, 85. DOI ADS

Kushwaha, U., Joshi, B., Cho, K.-S., Veronig, A., Tiwari, S.K., Mathew, S.K.: 2014, Impulsive Energy Release and Non-thermal Emission in a Confined M4.0 Flare Triggered by Rapidly Evolving Magnetic Structures. Astrophys. J. 791(1), 23. DOI ADS 
Lemen, J.R., Title, A.M., Akin, D.J., Boerner, P.F., Chou, C., Drake, J.F., Duncan, D.W., Edwards, C.G., Friedlaender, F.M., Heyman, G.F., Hurlburt, N.E., Katz, N.L., Kushner, G.D., Levay, M., Lindgren, R.W., Mathur, D.P., McFeaters, E.L., Mitchell, S., Rehse, R.A., Schrijver, C.J., Springer, L.A., Stern, R.A., Tarbell, T.D., Wuelser, J.-P., Wolfson, C.J., Yanari, C., Bookbinder, J.A., Cheimets, P.N., Caldwell, D., Deluca, E.E., Gates, R., Golub, L., Park, S., Podgorski, W.A., Bush, R.I., Scherrer, P.H., Gummin, M.A., Smith, P., Auker, G., Jerram, P., Pool, P., Soufli, R., Windt, D.L., Beardsley, S., Clapp, M., Lang, J., Waltham, N.: 2012, The Atmospheric Imaging Assembly (AIA) on the Solar Dynamics Observatory (SDO). Solar Phys. 275, 17. DOI ADS

Li, T., Zhang, J.: 2014, Slipping Magnetic Reconnection Triggering a Solar Eruption of a Triangle-shaped Flag Flux Rope. Astrophys. J. Lett. 791(1), L13. DOI ADS.

Li, T., Zhang, J.: 2015, Quasi-periodic Slipping Magnetic Reconnection During an X-class Solar Flare Observed by the Solar Dynamics Observatory and Interface Region Imaging Spectrograph. Astrophys. J. Lett. 804(1), L8. DOI ADS

Li, T., Yang, S., Zhang, Q., Hou, Y., Zhang, J.: 2018, Two Episodes of Magnetic Reconnections during a Confined Circular-ribbon Flare. Astrophys. J. 859, 122. DOI ADS

Lin, R.P., Dennis, B.R., Hurford, G.J., Smith, D.M., Zehnder, A., Harvey, P.R., Curtis, D.W., Pankow, D., Turin, P., Bester, M., Csillaghy, A., Lewis, M., Madden, N., van Beek, H.F., Appleby, M., Raudorf, T., McTiernan, J., Ramaty, R., Schmahl, E., Schwartz, R., Krucker, S., Abiad, R., Quinn, T., Berg, P., Hashii, M., Sterling, R., Jackson, R., Pratt, R., Campbell, R.D., Malone, D., Landis, D., Barrington-Leigh, C.P., Slassi-Sennou, S., Cork, C., Clark, D., Amato, D., Orwig, L., Boyle, R., Banks, I.S., Shirey, K., Tolbert, A.K., Zarro, D., Snow, F., Thomsen, K., Henneck, R., McHedlishvili, A., Ming, P., Fivian, M., Jordan, J., Wanner, R., Crubb, J., Preble, J., Matranga, M., Benz, A., Hudson, H., Canfield, R.C., Holman, G.D., Crannell, C., Kosugi, T., Emslie, A.G., Vilmer, N., Brown, J.C., Johns-Krull, C., Aschwanden, M., Metcalf, T., Conway, A.: 2002, The Reuven Ramaty High-Energy Solar Spectroscopic Imager (RHESSI). Solar Phys. 210, 3. DOI ADS.

Liu, R., Kliem, B., Titov, V.S., Chen, J., Wang, Y., Wang, H., Liu, C., Xu, Y., Wiegelmann, T.: 2016, Structure, Stability, and Evolution of Magnetic Flux Ropes from the Perspective of Magnetic Twist. Astrophys. J. 818(2), 148. DOI ADS

Liu, W., Berger, T.E., Title, A.M., Tarbell, T.D., Low, B.C.: 2011, Chromospheric Jet and Growing "Loop" Observed by Hinode: New Evidence of Fan-spine Magnetic Topology Resulting from Flux Emergence. Astrophys. J. 728(2), 103. DOI ADS

Masson, S., Pariat, E., Aulanier, G., Schrijver, C.J.: 2009, The Nature of Flare Ribbons in Coronal Null-Point Topology. Astrophys. J. 700, 559. DOI ADS.

Mitra, P.K., Joshi, B.: 2019, Preflare Processes, Flux Rope Activation, Large-scale Eruption, and Associated X-class Flare from the Active Region NOAA 11875. Astrophys. J. 884(1), 46. DOI ADS

Mitra, P.K., Joshi, B., Prasad, A.: 2020, Identification of Pre-flare Processes and Their Possible Role in Driving a Large-scale Flux Rope Eruption with Complex M-class Flare in the Active Region NOAA 12371. Solar Phys. 295(29). DOI

Moon, Y.-J., Chae, J., Choe, G.S., Wang, H., Park, Y.D., Cheng, C.Z.: 2004, Low Atmosphere Reconnections Associated with an Eruptive Solar Flare. Journal of Korean Astronomical Society 37(1), 41. DOI ADS

Nakajima, H., Dennis, B.R., Hoyng, P., Nelson, G., Kosugi, T., Kai, K.: 1985, Microwave and $\mathrm{X}$-ray observations of delayed brightenings at sites remote from the primary flare locations. Astrophys. J. 288, 806. DOI ADS

Pariat, E., Antiochos, S.K., DeVore, C.R.: 2010, Three-dimensional Modeling of Quasihomologous Solar Jets. Astrophys. J. 714, 1762. DOI ADS

Parker, E.N.: 1963, The Solar-Flare Phenomenon and the Theory of Reconnection and Annihiliation of Magnetic Fields. Astrophys. J. Suppl 8, 177. DOI. ADS

Pesnell, W.D., Thompson, B.J., Chamberlin, P.C.: 2012, The Solar Dynamics Observatory (SDO). Solar Phys. 275, 3. DOI ADS

Pontin, D., Galsgaard, K., Démoulin, P.: 2016, Why Are Flare Ribbons Associated with the Spines of Magnetic Null Points Generically Elongated? Solar Phys. 291(6), 1739. DOI ADS

Reid, H.A.S., Vilmer, N., Aulanier, G., Pariat, E.: 2012, X-ray and ultraviolet investigation into the magnetic connectivity of a solar flare. Astron. Astrophys. 547, A52. DOI ADS

Schou, J., Scherrer, P.H., Bush, R.I., Wachter, R., Couvidat, S., Rabello-Soares, M.C., Bogart, R.S., Hoeksema, J.T., Liu, Y., Duvall, T.L., Akin, D.J., Allard, B.A., Miles, J.W., Rairden, R., Shine, R.A., Tarbell, T.D., Title, A.M., Wolfson, C.J., Elmore, D.F., Norton, A.A., 
Tomczyk, S.: 2012, Design and Ground Calibration of the Helioseismic and Magnetic Imager (HMI) Instrument on the Solar Dynamics Observatory (SDO). Solar Phys. 275, 229. DOI ADS

Shibata, K., Magara, T.: 2011, Solar Flares: Magnetohydrodynamic Processes. Living Reviews in Solar Physics 8, 6. DOI ADS

Sturrock, P.A.: 1966, Model of the High-Energy Phase of Solar Flares. Nature 211, 695. DOI ADS

Sun, X., Hoeksema, J.T., Liu, Y., Aulanier, G., Su, Y., Hannah, I.G., Hock, R.A.: 2013, Hot Spine Loops and the Nature of a Late-phase Solar Flare. Astrophys. J. 778, 139. DOI] ADS

Svestka, Z.: 1986, On the varieties of solar flares. In: Neidig, D.F., Machado, M.E. (eds.) The Lower Atmosphere of Solar Flares, 332. ADS

Tang, F., Moore, R.L.: 1982, Remote Flare Brightenings and Type-Iii Reverse Slope Bursts. Solar Phys. 77(1-2), 263. DOI ADS.

Uddin, W., Schmieder, B., Chandra, R., Srivastava, A.K., Kumar, P., Bisht, S.: 2012, Observations of Multiple Surges Associated with Magnetic Activities in AR 10484 on 2003 October 25. Astrophys. J. 752(1), 70. DOI ADS

Veronig, A., Vršnak, B., Temmer, M., Hanslmeier, A.: 2002, Relative timing of solar flares observed at different wavelengths. Solar Phys. 208(2), 297. DOI. ADS

Wang, H., Liu, C.: 2012, Circular Ribbon Flares and Homologous Jets. Astrophys. J. 760, 101. DOI ADS

Wang, H., Liu, C., Ahn, K., Xu, Y., Jing, J., Deng, N., Huang, N., Liu, R., Kusano, K., Fleishman, G.D., Gary, D.E., Cao, W.: 2017, High-resolution observations of flare precursors in the low solar atmosphere. Nature Astronomy 1, 0085. DOI. ADS.

Wiegelmann, T., Inhester, B.: 2010, How to deal with measurement errors and lacking data in nonlinear force-free coronal magnetic field modelling? Astron. Astrophys. 516, A107. DOI ADS

Wiegelmann, T., Solanki, S.K., Yelles, L., Lagg, A.: 2007, Nonlinear Force-Free Magnetic Field Modelling For VIM On SO. In: Second Solar Orbiter Workshop, ESA Special Publication 641, 19. ADS

Xu, Z., Yang, K., Guo, Y., Zhao, J., Zhao, Z.J., Kashapova, L.: 2017, Homologous Circularribbon Flares Driven by Twisted Flux Emergence. Astrophys. J. 851, 30. DOI ADS

Zeng, Z., Chen, B., Ji, H., Goode, P.R., Cao, W.: 2016, Resolving the Fan-spine Reconnection Geometry of a Small-scale Chromospheric Jet Event with the New Solar Telescope. Astrophys. J. 819(1), L3. DOI ADS

Zhang, Q.M., Li, D., Huang, Y.: 2018, Imaging observations of chromospheric evaporation in a circular-ribbon flare. arXiv e-prints. ADS

Zheng, R., Chen, Y., Wang, B.: 2016, Slipping Magnetic Reconnections with Multiple Flare Ribbons during an X-class Solar Flare. Astrophys. J. 823(2), 136. DOI ADS.

Zhong, Z., Guo, Y., Ding, M.D., Fang, C., Hao, Q.: 2019, Transition from Circular-ribbon to Parallel-ribbon Flares Associated with a Bifurcated Magnetic Flux Rope. Astrophys. J. 871, 105. DOI ADS

Zuccarello, F.P., Chandra, R., Schmieder, B., Aulanier, G., Joshi, R.: 2017, Transition from eruptive to confined flares in the same active region. Astron. Astrophys. 601, A26. DOI] ADS 